\title{
Comparison of Pain Relief By Placing Regenerated Oxidized Cellulose Sheet Soaked in Bupivacaine And Port Site Infiltration With Bupivacaine Post-Laparoscopic Cholecystectomy: A Randomized Control Trial
}

\author{
Dr. Dharamdev D. ${ }^{1}$,Dr. Neil B. D'souza ${ }^{2}$ \\ ${ }^{1,2}$ (Department Of General Surgery, A. J. Institute Of Medical Sciences, India)
}

\begin{abstract}
:
Background: This study was conducted to determine postoperative pain control with use of Bupivacaine soaked sheet of Regenerated Oxidized Cellulose (ROC) in gallbladder (GB) bed and compared with port-site infiltration of bupivacaine after Laparoscopic Cholecystectomy $(L C)$.

Methods: Total 100 patients were divided in 3 groups. In Group A $(n=37)$ Bupivacaine soaked ROC was positioned in the gallbladder bed. In Group B $(n=28)$ Bupivacaine was infiltrated in trocar site before skin suturing and in Group $C(n=32)$ was control group. Nature of pain, either visceral, parietal, or shoulder, was assessed on Visual Analogue Scale(VAS) at 3, 6, and 24 hours after surgery.

Results: All patients (100\%) demonstrated different intensities of visceral and parietal pain in all groups. None of the patients had shoulder pain. Visceral pain was significantly less in Group A as compared to Group B and $C$ ( $p<0.05)$. Visceral pain score for Group A at 3, 6, and 24 hours assessment was 17.41\%, 21.76\%, and $19.02 \%$ which was significantly less than Group B $(37.69 \%, 29.39 \%$, and 33.21\%) and Group C $(51.63 \%$, 49.87\%, and 50.29\%). Parietal pain score for Group B at 3, 6, and 24 hours assessment was $17.35 \%, 16.67 \%$, and $17.02 \%$ which was less than Group A (24.57\%, 25.32\% and 26.34\%) and Group C (49.33\%, 49.27\%, and $51.22 \%)$ ( $p<0.05$ ). In Group A 10 patients (27\%) required postoperative analgesics within 10 hours of postoperative period. While in Group B 14 patients (50\%) and Group C 32 patients (100\%) required postoperative analgesia within 8 hours of postoperative period.

Conclusion: Bupivacaine with ROC in the gallbladder bed is safe, technically simple, increases postoperative comfort, and decreases postoperative analgesic requirement after LC as compared to trocar site infiltration.
\end{abstract}

Keywords: Bupivacaine, Laparoscopic Cholecystectomy, Intraperitoneal, Postoperative Pain, Analgesia

\section{Introduction}

Laparoscopic cholecystectomy has become the gold standard for treatment of benign gallbladder disease. Though laparoscopic surgery has a number of advantages over conventional open surgery, it is not a completely pain free procedure. Pain relief after cholecystectomy, either open or laparoscopic, remains a cause of concern. Pain after laparoscopic surgery though primarily visceral, often affecting the subdiaphragmatic region and often referred to right shoulder region, also has parietal components which occurs at the trocar site. Many factors such as humidity and temperature of gas, pressure of pneumoperitoneum during surgery, residual intraperitoneal gas at the end of surgery, duration of surgery and length of trocar incision, trauma caused by cholecystectomy itself, individual factors and sociocultural conditions play an important role in the causation of pain. Postoperative pain in turn affects postoperative morbidity, hospital stay, increased financial burden on the patient or the state due to increased duration of hospitalization and inability of the patient to return to his/her job on time. A number of studies have been done till date to try to assess the effectiveness of different measures to alleviate PO pain after LC. ${ }^{[1-22]}$ PO pain management has an important role in preventing the postoperative morbidity and its consequences. Pain management has not been standardized at mass medical centers in India and abroad and this is reflected in the number of different pain studies after LC available in literature. ${ }^{[1-22]}$ Till day, many different methods have been used with conflicting rates of success to diminish the intensity of PO pain after LC ${ }^{[1-9]}$. They include low pressure pneumoperitoneum, gasless technique of LC, use of warm carbon dioxide, peritoneal wash with saline solution, strict surgical technique, perfect hemostasis, trocar site infiltration of anaesthetic drugs, instillation ofsubdiaphragmatic region with anaesthetic/analgesic drugs, or use of nonsteriodal anti-inflammatory drugs or dexamethasone. ${ }^{[1-9]}$

To gain a major insight into the effectiveness of intraperitoneal use of $0.5 \%$ bupivacaine soaked sheet of regenerated oxidized cellulose and port site infiltration of bupivacaine in PO pain relief after laparoscopic cholecystectomy, the present study was carried out in the Department of General Surgery, A. J. Institute of Medical Sciences. We hope to establish conclusive evidence about the efficacy of both the procedures and whether one was superior to the other for total pain relief. 


\section{Methods}

A prospective, randomized study of 100 patients of benign gallbladder disease in whom laparoscopic cholecystectomy was conducted in the Department of General Surgery at A. J. Institute of Medical Sciences, Mangalore during the period of 16 months from June 2015 to September 2016. In all the cases a detailed history, physical examination, and investigations were done. Inclusion criteria was age above 18 years, ASA grade 1 or 2 (American Society of Anaesthesiology), gallbladder calculi or polyp with no evidence of acute cholecystitis at the time of surgery. Exclusion criteria was age below 18 years, ASA grade more than 2 (American Society of Anaesthesiology), pregnant females, acute cholecystitis, choledocholithiasis, conversion of LC to open cholecystectomy, and patients with past history of major abdominal surgery. After taking informed and written consent, patients were randomized in 3 groups. Randomization was done with prepared envelope. The day before operation an independent hospital staff randomly opened an envelope with a card inside. Patients were randomized to their respective card group, either 'A', 'B', or ' $C$ '. Patients were blinded, they did not know into which group they were being allotted.

Group A: Bupivacaine soaked sheet of regenerated oxidized cellulose positioned in the gallbladder bed after removal of gallbladder and before evacuation of pneumoperitoneum. Group B: Bupivacaine infiltrated in muscular fasciae of trocar before skin suturing. Group C: Control group, not using any local anaesthetic drug. For the patients undergoing laparoscopic cholecystectomy, ultrasonography, X Ray chest, ECG, routine haemogram, liver function tests, random blood sugar, blood urea, serum creatinine, physician and preanaestheticcheck up for fitness for surgery was done and Inj. Cefotaxime was given preoperatively just before the induction of anaesthesia.

In all patients premedication of Inj. Glycopyrolate (5microgram/kg), Inj. Tramadol (1mg/kg), Inj. Ranitidine (50mg) and Inj. Ondansetron $(4 \mathrm{mg})$ was given 15 minutes before induction intravenously. Induction was achieved with Inj. Thiopentone $(7 \mathrm{mg} / \mathrm{kg})$ IV and endotracheal intubation was done after muscle relaxation with Succinylcholine $(2 \mathrm{mg} / \mathrm{kg})$. Anaesthesia was maintained with Oxygen and Nitrous oxide (50:50) and Sevoflurane. Ventilation was adjusted to maintain end-tidal pressure of $\mathrm{CO}_{2}$ to $<40 \mathrm{mmHg}$. Intraoperative intravenous fluid supplementation was given, either Ringers Lactate, Dextrose Normal Saline, 5\% Dextrose, or Normal Saline. Intraoperative ECG, NIBP, $\mathrm{SPO}_{2}$, and $\mathrm{ET} \mathrm{CO}_{2}$ monitoring was done.

LC was performed by creating pneumoperitoneum with Veress needle at the rate of $2 \mathrm{~L} / \mathrm{min}$ with four ports (two $10 \mathrm{~mm}$ and two $5 \mathrm{~mm}$ ). The gas pressure was allowed to build up to $12-14 \mathrm{mmHg}$ before introducing first blind trocar and was maintained throughout the surgery. After removal of gallbladder perfect hemostasis was achieved and gallbladder bed was thoroughly washed with normal saline. Gallbladder specimen was sent to Pathology Department for histopathological examination. If necessary drain was inserted through lateral trocar and positioned in sub-hepatic region. In Group A after removal of GB, 0.5\% bupivacaine soaked ROC was placed in GB bed ${ }^{[3,4]}$ (Fig. 1). Remaining bupivacaine was injected through suction cannula over ROC in GB bed.

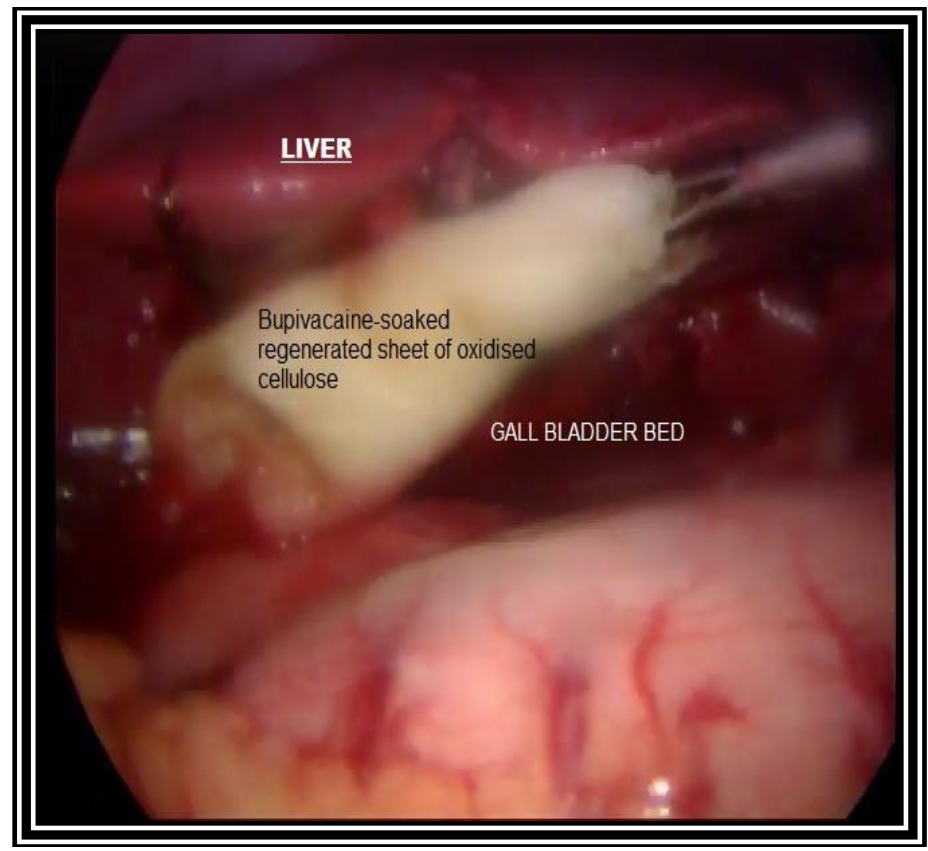

Figure 1. Bupivacaine soaked sheet of regenerated oxidized cellulose placed in GB bed after removal of GB (Group A). 


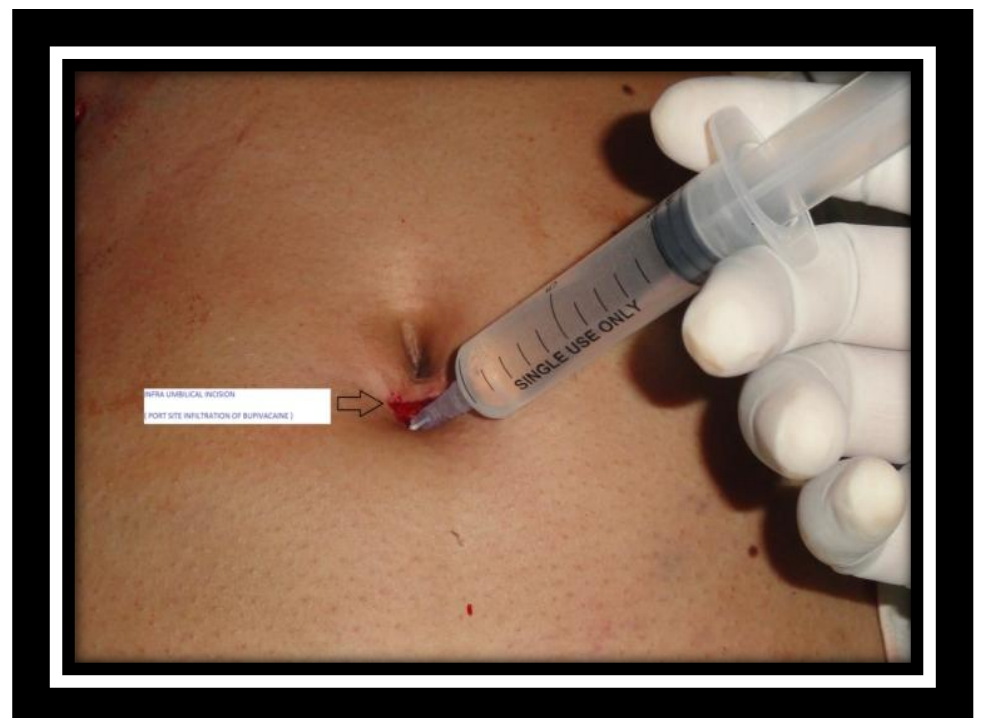

Figure 2. Treocar site infiltration of bupivacaine (Group B).

In Group B, before skin suturing bupivacaine $2 \mathrm{mg} / \mathrm{kg}$ was infiltrated into muscular fasciae at trocar site (Fig. 2) and in Group C no local anaesthetic drug was given. Analgesic injection Tramadol $1 \mathrm{mg} / \mathrm{kg}$ was given on patient's demand for postoperative pain relief.Postoperatively, assessment of nature of pain either visceral, parietal, or shoulder pain was done and its intensity was recorded on VAS (Visual Analogue Scale) at 3 hours, 6 hours, and 24 hours of surgery. VAS was a $100 \mathrm{~mm}$ horizontal scale representing varying intensity of pain with endpoints labelled as "no pain" and "severe pain". Deep seated abdominal pain felt by the patient in the right hypochondrium was considered as visceral pain. Pain at trocar site was considered as parietal pain. Referred pain to shoulder was considered as shoulder pain.First request for analgesics, vomiting, duration of surgery, intraperitoneal drain was kept or not, length of hospital stay, any other postoperative complications was recorded.

\section{Statistical Analysis}

Was done using ANOVA for comparison between Groups A, B, and C. Independent T test was applied to compare the mean score difference within the study groups (A-B, B-C, and A-C), 'p' value of $<0.05$ was considered to be statistically significant.

\section{Results}

A total of 100 (73 females, 27 males) patients of benign gallbladder disease in whom LC was conducted in the Department of General Surgery at A. J. Institute of Medical Sciences during the period of 16 months from June 2015 to September 2016, were enrolled out of which 3 patients were excluded from the study due to laparoscopic to open conversion because of intraoperative bleeding (1) and gallbladder adhesion (2). So a total of 97 patients (70 females, 27 males) were included in this study. They were randomized in 3 groups $(A=37, B=28, C=32)$. There was no significant difference in age, duration of surgery, ASA, weight, hospital stay, number of trocars (Table 1). All patients demonstrated different intensities of visceral and parietal pain. However none of the patients developed shoulder pain in the postoperative pain in either group. In Group A total 10 patients out of 37 required intraperitoneal drain placement. In Group B it was 6 out of 28 and in Group $\mathrm{C}$ it was 7 out of 32 .

Table 1: Demographic and operative details of analyzed data

\begin{tabular}{|l|l|l|l|l|l|l|l|l|}
\hline Group & Patients & Sex(M/F) & Age(Y) & Surgery(Min) & $\begin{array}{l}\text { ASA } \\
(\mathrm{I} / \mathrm{II})\end{array}$ & Weight(kg) & $\begin{array}{l}\text { Hospital } \\
\text { Stay(Days) }\end{array}$ & Trocars \\
\hline A & 37 & $11 / 26$ & $36.9 \pm 8.82$ & $75.72 \pm 18.22$ & $30 / 7$ & $52.67 \pm 5.12$ & $3 \pm 2$ & 4 \\
\hline B & 28 & $7 / 21$ & $33.12 \pm 8.86$ & $77.66 \pm 18.12$ & $24 / 4$ & $51.36 \pm 5.88$ & $3 \pm 2$ & 4 \\
\hline C & 32 & $9 / 23$ & $38.32 \pm 9.12$ & $80.12 \pm 21.32$ & $23 / 9$ & $55.32 \pm 5.05$ & $3 \pm 2$ & 4 \\
\hline
\end{tabular}

In Group A 10 patients out of 37 required analgesic injection Tramadol postoperatively. Mean time was 10 hours for first request and 24 hours for second request in PO period. Rest 27 patients never asked for analgesics in PO period. In Group B 14 out of 28 patients required analgesics after 8 hours (first request) and after 24 hours (second request) in PO period. 14 patients in Group B never asked for analgesics. In Group C all 32 out of 32 patients required analgesics after 8 hours and 24 hours PO.In Group A 10 patients required PO 
analgesics, out of those patients 10 had intra-peritoneal drain. In Group A all patients (100\%) with PO intraperitoneal drain required analgesics. 27 patients (72\%) without drain never asked for PO analgesics. In Group B 17 patients $(60 \%)$ required PO analgesics out of 28 patients. Out of those 17 patients 8 had intraperitoneal drain. 11 patients (39\%) never asked for analgesics in Group B. In Group C 32 out of 32 patients (100\%) required PO analgesics. Total 8 patients $(25 \%)$ had PO intraperitoneal drain.

Table 2: Comparison between various groups of post-op analgesics (tramadol injection) requirement

\begin{tabular}{|l|l|l|l|}
\hline & Group A and B & Group A and C & Group B and C \\
\hline 't' value & 1.032 & 5.82 & 4.24 \\
\hline 'p' value & 0.3102 & 0.0001 & 0.0002 \\
\hline Significance ('p' value $<0.05)$ & Not significant & Significant & Significant \\
\hline
\end{tabular}

In Group A 10 patients (27\%) required PO analgesia within 10 hours in PO period while in Group B 17 patients (60\%) and Group C 32 patients (100\%) required PO analgesics within 8 hours in PO period (Table 2). On comparing Group A with Group C for PO analgesics requirement, $\mathrm{PO}$ analgesic requirement was less in Group A and 'p' value was highly significant ('p'-0.0001<0.05). On comparing Group B with Group C for the same, PO analgesics requirement was less in Group B with significant ' $p$ ' value (' $p$ ' $=0.0002<0.05)$. 'p' value was not significant between Group A and B ('p'=0.3102>0.05).

Table 3: Statistical analysis of VAS

\begin{tabular}{|l|l|l|l|}
\hline & Group & Total patient & Pain Score \\
\hline Visceral Pain & A & 37 & 17.41 \\
\hline 3 hours & B & 28 & 37.69 \\
\hline & C & 32 & 51.63 \\
\hline 6 hours & A & 37 & 21.76 \\
\hline & B & 28 & 29.39 \\
\hline 24 hours & C & 32 & 49.87 \\
\hline & A & 37 & 19.02 \\
\hline & B & 28 & 33.21 \\
\hline Parietal Pain & C & 32 & 50.29 \\
\hline 3 hours & A & 37 & 24.57 \\
\hline & B & 28 & 17.35 \\
\hline 6 hours & C & 32 & 49.33 \\
\hline & A & 37 & 29.32 \\
\hline & B & 28 & 16.67 \\
\hline 24 hours & C & 32 & 49.27 \\
\hline & A & 37 & 26.34 \\
\hline & B & 28 & 17.02 \\
\hline & C & 32 & 51.22 \\
\hline
\end{tabular}

Above table shows comparison between all 3 groups

Table 4: Comparison between study groups (A, B, and C) using analysis of variance (ANOVA)

\begin{tabular}{|l|l|}
\hline Pain & $\mathrm{p}$ value \\
\hline Visceral pain 3 hours & 0.001 \\
\hline Parietal pain 3 hours & 0.02 \\
\hline Visceral pain 6 hours & 0.001 \\
\hline Parietal pain 6 hours & 0.001 \\
\hline Visceral pain 24 hours & 0.03 \\
\hline Parietal pain 24 hours & 0.001 \\
\hline
\end{tabular}

On comparison between Group A and Group B, data shows that the mean visceral pain score of VAS was less at all assessment (3hrs, 6hrs and 24hrs) for Group A as compared to Group B (Table3). Mean parietal pain score of VAS was less for Group B as compared to Group A at all assessments (3hrs, 6hrs and 24hrs) (Table 3) with significant 'p' value ('p'<0.05) (Table4).

Table 5: 'p'-values between Group A and Group B (by independent T test)

\begin{tabular}{|l|l|l|l|l|l|l|}
\hline & $\mathrm{V}_{3}$ & $\mathrm{~V}_{6}$ & $\mathrm{~V}_{24}$ & $\mathrm{P}_{3}$ & $\mathrm{P}_{6}$ & $\mathrm{P}_{24}$ \\
\hline $\begin{array}{l}\text { Mean Difference } \\
\text { (MD) }\end{array}$ & -20.28 & -7.63 & -14.19 & -11.83 & -12.65 & -9.32 \\
\hline 'p' & 0.02 & 0.02 & 0.01 & 0.03 & 0.03 & 0.01 \\
\hline
\end{tabular}

(V=visceral, $\mathrm{P}=$ parietal, $3=3 \mathrm{hrs}, 6=6 \mathrm{hrs}$, ' $\mathrm{p}$ ' value $(0.05=$ statistically significant $)$

On comparing between Group A and Group C both mean visceral pain score of VAS as well as mean parietal pain score of VAS was less in Group A as compared to Group C at 3 hours, 6 hours, and 24 hours with significant 'p' value ('p'<0.05) (Table 3 and Table 5). 
Table 6: 'pe'-values between Group A and Group C (by independent T test)

\begin{tabular}{|l|l|l|l|l|l|l|}
\hline & $\mathrm{V}_{3}$ & $\mathrm{~V}_{6}$ & $\mathrm{~V}_{24}$ & $\mathrm{P}_{3}$ & $\mathrm{P}_{6}$ & $\mathrm{P}_{24}$ \\
\hline $\begin{array}{l}\text { Mean Difference } \\
\text { (MD) }\end{array}$ & -34.22 & -28.11 & -31.27 & -34.42 & -32.60 & -34.20 \\
\hline 'p' & 0.001 & 0.001 & 0.02 & 0.001 & 0.02 & 0.01 \\
\hline
\end{tabular}

( $\mathrm{V}=$ visceral, $\mathrm{P}=$ parietal, $3=3 \mathrm{hrs}, 6=6 \mathrm{hrs}$, 'p' value $(0.05=$ statistically significant $)$

Table 7: ' $\mathrm{p}$ '-values between Group B and Group C (by independent T test)

\begin{tabular}{|l|l|l|l|l|l|l|}
\hline & $\mathrm{V}_{3}$ & $\mathrm{~V}_{6}$ & $\mathrm{~V}_{24}$ & $\mathrm{P}_{3}$ & $\mathrm{P}_{6}$ & $\mathrm{P}_{24}$ \\
\hline $\begin{array}{l}\text { Mean Difference } \\
\text { (MD) }\end{array}$ & -13.94 & -20.48 & -17.08 & -23.31 & -19.95 & -24.88 \\
\hline 'p' & 0.001 & 0.001 & 0.001 & 0.01 & 0.001 & 0.001 \\
\hline
\end{tabular}

( $\mathrm{V}=$ visceral, $\mathrm{P}=$ parietal, $3=3 \mathrm{hrs}, 6=6 \mathrm{hrs}$, ' $\mathrm{p}$ ' value $(0.05=$ statistically significant $)$

On comparison between Group B and Group C, data shows both mean visceral (VAS) and mean parietal (VAS) pain score was less in Group B as compared to Group C at all assessments (3 hours, 6 hours, 24 hours) with significant ' $p$ ' value ('p'<0.05) (Table 3 and Table 6).

\section{Discussion}

PO pain control after LC remains a major cause of concern. The fact that in many hospitals LC is performed as a daycare procedure emphasizes the need for early and appropriate PO pain relief so that the patient has a painless discharge postoperatively. There was increased predominance of female patients in all the studies including the present study which is natural because of high incidence of gallstone disease in female patients. Determining the sex incidence in any pain study is important because women report more pain than men.

In present study none of the patients had shoulder pain in postoperative period. This was at odds with results of other studies by Chundrigar $\mathrm{T}$ et al ${ }^{[8]}-12 \%$, Feroci $\mathrm{F}$ et al ${ }^{[3]}-44 \%$ and Verma $\mathrm{G} \mathrm{R}$ et al ${ }^{[4]}-14 \%$. It is difficult to attribute any particular cause to the absence of shoulder pain in this study. It is postulated that shoulder pain is due to $\mathrm{CO}_{2}$ gas trapped beneath the right hemidiaphragm after deflation of abdomen. We were meticulous in postoperative deflation after LC. This may partly explain low incidence.

Visceral and parietal pain accounted for most of the pain in early PO period (3 hours and 6 hours) in this study. Visceral pain score $(51.63 \%$ and $49.87 \%)$ and parietal pain score $(49.33 \%$ and $49.27 \%)$ were highest at 3 hours and 6 hours assessment in control Group C (Table 3). Though Joris et al ${ }^{[11]}$ and Verma G R ${ }^{[4]}$ have concluded this study that visceral pain is predominant, we were unable to concur with them as both the scores were similar.

In the present study we used bupivacaine soaked in regenerated oxidized cellulose at the optimal dose of $2 \mathrm{mg} / \mathrm{kg}$ to increase contact time of bupivacaine at gallbladder bed so as to increase absorption and get maximum PO pain relief. The peak serum level of intraperitoneal bupivacaine was reached 20-30 minutes after application and lasts for 2-24 hours after surgery. It was evident that the methods in this study significantly reduced visceral pain when compared to control group as was seen in trials by Feroci $\mathrm{F}^{[3]}$ and Verma $\mathrm{G} \mathrm{R}$ et al [4] . Visceral pain was significantly low in Group A $(17.41 \%$ and $21.76 \%)$ as compared to Group B (37.69\% and $29.39 \%$ ) and Group C (51.63\% and 49.87\%) at 3 hours and 6 hours assessment (Table 3). Intraperitoneal use of this drug without using regenerated oxidized cellulose leads to reduced contact of this drug to gallbladder bed (surgical trauma site) ${ }^{[11,15]}$ and decreased absorption time of bupivacaine leading to increased PO analgesic demand by the patients.

It is also important to point out that a dose response relationship to the use of intraperitonealanaesthetics has been observed as can be seen from the results of one of the groups in the study by Verma $G$ R et al ${ }^{[4]}$ in which half the dose of bupivacaine was instilled over ROC in liver bed and the other half infiltrated in the trocar site. In these patients parietal and visceral pain was similar to control group possibly due to inadequate dosage. Precisely to avoid such a possibility the study was designed in such a way so as to avoid the possibility of inadequate dosing. In the present study we have not used bupivacaine at two different sites at the same time as this divides the effect of drugs at its optimal dose $(2 \mathrm{mg} / \mathrm{kg})$.

Use of intraperitoneal drain PO after LC leads to drainage of bupivacaine through the drain and increases PO analgesic requirement. If intraperitoneal drain is required after LC use of intraperitoneal bupivacaine is avoided. In Group B when bupivacaine was infiltrated to trocar site it significantly reduced the parietal pain (17.35\% and 16.67\%) as compared to Group C (49.33\% and $49.27 \%)$ and Group A (24.57\% and $29.32 \%$ ) (Table 3 ) in early PO period at 3 hours and 6 hours ('p' value $<0.05$ ). Parietal pain comes near to the baseline $(\mathrm{O})$ within 24 hours in Group B while in other two groups they remain above the baseline. These findings support the findings of two previous studies where local infiltration of bupivacaine at its optimal dosage at trocar sites reduced parietal pain ${ }^{[3,4]}$ 
Bupivacaine in either form of administration at its optimal dose reduces PO analgesic requirement as compared to control group. All patients in control group (Group C) required analgesics within 8 hours as compared to $27 \%$ for Group A and $50 \%$ for Group B. There was a clinically significant decrease in the mean analgesic requirement in Group A and B as compared to the control Group C. These findings are comparable to the study by Feroci $\mathrm{F}$ et al ${ }^{[3]}$.

\section{Conclusion}

Use of $0.5 \%$ bupivacaine soaked sheet of ROC in the gallbladder after LC is effective than trocar site infiltration to control PO pain and reduces PO analgesic requirement. Its use should be avoided in patients with intraperitoneal drain. Visceral and parietal pain are predominant in postoperative period after LC caused by surgical trauma at gallbladder bed and abdominal incision. Prevalence of shoulder tip pain in this study was not observed which could be attributable to meticulous deflation of pneumoperitoneum.

\section{References}

[1]. Slim K, Bousquet J, Kwiatkowski F et al (1999) Effect of CO2 gas warming on pain after laparoscopicsurgery: a randomized double-blind controlled trial. SurgEndosc 13:1110-1114

[2]. Saad S, Minor I, Mohri T et al (2000) The clinical impact of warmedinsufflation carbon dioxide gas for laparoscopic cholecystectomy. SurgEndosc 14:787-790.

[3]. Feroci F, Kroning KC, Scatizzi M(2008),Effectiveness for pain after laparoscopic cholecystectomy of $0.5 \%$ bupivacaine-soaked Tabotampplaced in the gallbladder bed, Surg Endosc:10: 0301-6.

[4]. Verma GR, Lyngdoh TS, Kaman L et al (2006) Placement of 0.5\% bupivacaine-soaked Surgicel in the gallbladder bed is effective for pain after laparoscopic cholecystectomy. SurgEndosc 20:1560-1564.

[5]. Sarli L, CostiR,Sansebastiano G et al (2000) Prospective randomizedtrial of low-pressure pneumoperitoneum for reduction of shoulder-tippain following laparoscopy. Br J Surg 87:1161-1165.

[6]. Barczynski M, Herman RM (2004) Low-pressure pneumoperitoneumcombined with intraperitoneal saline washout for reduction of pain after laparoscopic cholecystectomy: a prospective randomized study. SurgEndosc 18:1368-1373.

[7]. Lepner U, Goroshina J, Samaru“tel J (2003) Postoperative pain relief after laparoscopic cholecystectomy: a randomised prospective doubleblind clinical trial. Scand J Surg 92:121-124.

[8]. Chundrigar T, Hedges AR, Morris R et al (1993) Intraperitoneal bupivacaine for effective pain relief after laparoscopic cholecystectomy. AnnR CollSurgEng175:437-439.

[9]. Sarac AM, Aktan AO, Baykan N et al (1996) The effect and timing of local anesthesia in laparoscopic cholecystectomy. SurgLaparoscEndosc 6:362-366.

[10]. AlkhamesiNA,PeckDH,LomaxAandDarziAW (2006), Intraperitonealaerosolization of bupivacaine reduces postoperative pain in laparoscopic surgery: a randomized prospective controlled double-blindedclinical trial, SurgEndosc:21:4,602-606.

[11]. Joris J, Thiry E, Paris $\mathrm{P}$ et al (1995) Pain after laparoscopic cholecystectomy: characteristics and effect of intraperitonealbupivacaine. AnesthAnalg 81:379-384.

[12]. Wills VL, Hunt DR (2000) Pain after laparoscopic cholecystectomy.Br JSurg 87: 273-284.

[13]. Barczynski M, Konturek A, Herman RM,(2005) Superiority of preemptive analgesia with intraperitoneal instillation of bupivacaine before rather than after the creation of pneumoperitoneum for laparoscopic cholecystectomy: a randomized, double-blind, placebocontrolledstudy: SurgEndosc: 20: 1088-1093.

[14]. Raetzell M, Maier. C, Schrijder.D, and Wulf.H,(1995), IntraperitonealApplication of Bupivacaine During Laparoscopic Cholecystectomy-Riskor Benefit?: Anesth Analg;81:967-72.

[15]. Pascqualucci A, De Angelis V, Contardo R, Colo F, TerrosuG,Donini A, Pasetto A, Bresadola F (1996) Preemptive analgesia: A randomized, double-blind, placebo-controlled study. Anesthesiology 85: 11-20.

[16]. Mouton WG, Bessell JR, Pfitzner J et al (1999) A randomized controlledtrial to determine the effects of humidified carbondioxideinsufflationduringthoracoscopy. SurgEndosc 13:382-385.

[17]. Schulte-Steinberg H, Weninger E, Jokisch D et al (1995) Intraperitoneal versus interpleural morphine or bupivacaine for pain after laparoscopic cholecystectomy. Anesthesiology 82:634-640.

[18]. Bisgaard T, Kehlet H, Rosenberg J (2001) Pain and convalescence afterlaparoscopic cholecystectomy. Eur J Surg 167:84-96.

[19]. Wills VL, Hunt DR (2000) Pain after laparoscopic cholecystectomy. Br J Surg 87:273-284.

[20]. Kehlet H, Gray AW, Bonnet F et al (2005) A procedure-specific systematic review and consensus recommendations for postoperative analgesia following laparoscopic cholecystectomy. SurgEndosc 19:1396-1415.

[21]. Lepner U, Goroshina J, Samaru tel J (2003) Postoperative pain relief after laparoscopic cholecystectomy: a randomised prospective doubleblind clinical trial.Scand J Surg 92:121-124

[22]. Elfberg BA, Sjo“vall-Mjo“berg S (2000) Intraperitoneal bupivacaine does not effectively reduce pain after laparoscopic cholecystectomy: a randomized, placebo-controlled and double blind study. SurgLaparoscEndoscPercutan Tech 10:357-359 